\title{
Writing an Effective Review Article
}

\author{
Christine M. Murphy
}

Published online: 3 May 2012

(C) American College of Medical Toxicology 2012

Review articles present an excellent overview of material pertaining to a specific topic or clinical question. There are different formats used to write review articles, and within academic medicine, a debate over the best format for review articles exists. The focus of a review may dictate the format; however, regardless of the format chosen, a structured approach is critical for crafting a comprehensive review with limited bias.

There are two general categories or types of review articles: narrative and systematic. Narrative review articles provide an overview of a broad spectrum of material in an easily readable format. Systematic review articles traditionally have employed very detailed and extensive searches for literature pertaining to a given topic with a critical review of the literature selected $[1,2]$. Many people consider the systematic review the gold standard of reviews because of the rigorous methods employed in evaluating existing literature. The thought behind this being that the more stringent the methods and critique are, the less author bias is introduced.

Systematic reviews can be further broken down into qualitative and quantitative reviews (see Table 1 for examples). In both qualitative and quantitative systematic reviews, authors attempt to obtain all relevant primary research, search multiple databases, perform manual searches of relevant referenced material, contact authors of abstracts containing unpublished material, systematically review and rate the literature, and critically evaluate the studies included in the review [2]. The

C. M. Murphy $(\bowtie)$

Department of Emergency Medicine, Carolinas Medical Center, PO Box 32861, MEB 3rd Floor,

Charlotte, NC 28232, USA

e-mail: christine.murphy66@gmail.com key difference is that quantitative reviews attempt to pool data between studies and statistically compare them (ex: metaanalysis).

Realistically, some topics are just better covered as a narrative review-historical reviews or reviews that are based heavily on historical information and studies do not fit well into systematic review formats. Additionally, within toxicology, research on a given topic may include multiple animal studies with varying animal models or clinical/in vivo evidence which may be limited to case series or case reports that do not fit well with the systematic review format. If the data are best presented in the narrative format, there are multiple ways to strengthen the review by incorporating elements of the systematic review to assist in limiting author bias (Table 1). First and foremost, provide full disclosure of the search methods used to allow for greater transparency and reproducibility. Use at minimum two databases for searching, as not all journals are indexed in MEDLINE and this will help to prevent accidental omission of key articles [2]. Finally, articles should convey a clear message.

Table 1 Examples of systematic and narrative reviews

\begin{tabular}{lll}
\hline Author & Review design & Reference \\
\hline Dougherty et al. & Qualitative & {$[3]$} \\
Cumpston & Qualitative & {$[4]$} \\
Schaeffer et al. & Quantitative & {$[5]$} \\
Phillips et al. & Narrative & {$[6]$} \\
Nelson et al. & Narrative & {$[7]$} \\
Grundlingh et al. & Narrative & {$[8]$} \\
\hline
\end{tabular}


Review articles provide a great deal of information to readers. While editors prefer systematic over narrative reviews, both have strengths. Striking a balance between the two is necessary for topics that may lend themselves to more of a narrative style.

Guidelines for preparation of review articles for JMT:

1. Focused and comprehensive reviews are expected to be critical assessments of literature and data sources pertaining to clinical topics, emphasizing factors such as etiology, diagnosis, prognosis, therapy, or prevention.

2. Systematic and narrative reviews are accepted, although a systematic approach should be used whenever possible.

3. All articles or data sources should be searched for and selected systematically for inclusion and critically evaluated. The specific type of study or analysis, population, intervention, exposure, tests, or outcomes should be described for each article or data source.

4. The title should indicate to the reader this is a review (ex: "Clearing the air: a review of the effects of particulate matter air pollution on human health" [9])

5. Review articles that address a specific clinical question should include a short case vignette or paragraph (no more than 100 words) describing the clinical question in the Introduction.

6. The search and selection process of reviewed material must be described in the manuscript for all review types in the Methods section. At a minimum, this should include databases and years searched, and key words used, as well as inclusion and exclusion criteria. Presentation of this information in written or tabular form within this section is acceptable.
7. Other sections should be included when appropriate and depending on the review topic (ex: Pathophysiology, Pharmacokinetics, Clinical Presentation, Diagnosis, Management, Prevention).

Conflicts of Interest There are no conflicts to declare.

\section{References}

1. Collins JA, Fauser B (2005) Balancing the strengths of systematic and narrative reviews. Hum Reprod Update 11:103-104

2. Green BN, Johnson CD, Adams A (2001) Writing narrative literature reviews for peer-reviewed journals: secrets of the trade. J Sports Chiropract Rehabil 15:5-19

3. Dougherty PP, Klein-Schwartz W (2010) Octreotide's role in the management of sulfonylurea-induced hypoglycemia. J Med Toxicol 6:199-206

4. Cumpston KL (2011) Is there a role for fasciotomy in Crotalinae envenomations in North America? Clin Toxicol 49:351-365

5. Schaeffer TH, Khatri V, Reifler LM, Lavonas EJ (2012) Incidence of immediate hypersensitivity reaction and serum sickness following administration of Crotalidae Polyvalent Immune Fab antivenom: a meta-analysis. Acad Emerg Med 19:121-131

6. Phillips S, Palmer RB, Brody A (2008) Epidemiology, toxicokinetics, and health effects of methyl tert-butyl ether (MTBE). J Med Toxicol 4:115-126

7. Nelson L, Schwaner R (2009) Transdermal fentanyl: pharmacology and toxicology. J Med Toxicol 5:230-241

8. Grundlingh J, Dargan PI, El-Zanfaly M, Wood DM (2011) 2,4Dinitrophenol (DNP): a weight loss agent with significant acute toxicity and risk of death. J Med Toxicol 7:205-212

9. Anderson JO, Thundiyil JG, Stolbach A (2012) Clearing the air: a review of the effects of particulate matter air pollution on human health. J Med Toxicol. doi:10.1007/s13181-011-0203-1 